\title{
A characterization of $\omega$-limit sets for piecewise monotone maps of the interval
}

by

\author{
Andrew D. Barwell (Birmingham)
}

\begin{abstract}
For a piecewise monotone map $f$ on a compact interval $I$, we characterize the $\omega$-limit sets that are bounded away from the post-critical points of $f$. If the pre-critical points of $f$ are dense, for example when $f$ is locally eventually onto, and $\Lambda \subset I$ is closed, invariant and contains no post-critical point, then $\Lambda$ is the $\omega$-limit set of a point in $I$ if and only if $\Lambda$ is internally chain transitive in the sense of Hirsch, Smith and Zhao; the proof relies upon symbolic dynamics. By identifying points of $\omega$-limit sets via their limit-itineraries, we offer simple examples which show that internal chain transitivity does not characterize $\omega$-limit sets for interval maps in general.
\end{abstract}

1. Introduction. Given a continuous map $f$ on a compact metric space $X$ with metric $d$, the $\omega$-limit set of a point $x \in X, \omega(x, f)=\bigcap_{n \in \mathbb{N}} \overline{\left\{f^{k}(x): k \geq n\right\}}$, is the set of accumulation points of the orbit $\operatorname{orb}(x, f)$ of $x$. The asymptotic behaviour of $f$ at the point $x$ can be ascertained by studying the structure of the $\omega$-limit set at that point. The structure of $\omega$-limit sets for maps on a compact interval has been investigated by many authors, including Block and Coppel [3] and Hirsch et al. [9], and characterizations of such sets have been given by Balibrea and La Paz [1], Blokh et al. 4] and by this author with Good, Knight and Raines [2]. In this paper we give a characterization based on the metric property of internal chain transitivity.

Given $\epsilon>0$, a sequence of points $\left\{x_{0}, \ldots, x_{n}\right\}$ is called an $\epsilon$-pseudo-orbit if $d\left(f\left(x_{i}\right), x_{i+1}\right)<\epsilon$ for $i=0, \ldots, n-1$.

Definition 1.1. A subset $\Lambda \subset X$ is called internally chain transitive if for every pair of points $x, y \in \Lambda$ and every $\epsilon>0$ there is an $\epsilon$-pseudo-orbit $\left\{x_{0}=x, x_{1}, \ldots, x_{n}=y\right\} \subset \Lambda$.

It is well known that for compact metric spaces, $\omega(x, f)$ is closed, invariant (by which we mean that $f(\omega(x, f))=\omega(x, f))$ and non-empty, and

2010 Mathematics Subject Classification: 37B10, 37E05, 54C05, 54H20.

Key words and phrases: omega-limit set, piecewise monotone map, symbolic dynamics, kneading theory. 
in [9] it is shown that it is also internally chain transitive. In [2] we show that internal chain transitivity characterizes $\omega$-limit sets for shifts of finite type, and also certain $\omega$-limit sets for tent maps with large enough gradient. The main result in this paper (Theorem 4.3) generalizes the characterization in [2] of $\omega$-limit sets of tent maps to a characterization of $\omega$-limit sets for piecewise monotone maps of the interval. Examples 2.7 and 5.5 show that internal chain transitivity does not characterize $\omega$-limit sets for maps of the interval in general.

The characterizations of $\omega$-limit sets for interval maps found in [1] and [4] contain definitions relating specifically to interval maps; in this paper we rely largely upon symbolic dynamics for our analysis, which allows us to give our characterization requiring internal chain transitivity only. Furthermore, we extend a well-known test using the limit-itineraries of points in the interval to check whether any point is in the $\omega$-limit set of any other point (Theorem 5.4.

2. Symbolic dynamics and kneading theory. Consider a finite alphabet $\Omega$ and the space of infinite sequences $\Omega^{\mathbb{N}}=\left\{\left(s_{0} s_{1} \ldots\right): s_{i} \in \Omega\right\}$. For $s=\left(s_{i}\right)$ and $t=\left(t_{i}\right)$ in $\Omega^{\mathbb{N}}$ let $\left.s\right|_{n}=\left(s_{0} \ldots s_{n}\right)$, and if $s \uparrow_{k-1}=t \uparrow_{k-1}$ but $s_{k} \neq t_{k}$ we say the discrepancy between $s$ and $t$ is $k$. Define a metric $d$ on $\Omega^{\mathbb{N}}$ by letting $d(s, t)=1 / 2^{k}$, where $k$ is the discrepancy between $s$ and $t$. This metric gives rise to the Tikhonov product topology on $\Omega^{\mathbb{N}}$; $\Omega$ is compact so $\Omega^{\mathbb{N}}$ is also compact. Define the shift map $\sigma: \Omega^{\mathbb{N}} \rightarrow \Omega^{\mathbb{N}}$ by $\sigma\left(s_{0} s_{1} s_{2} \ldots\right)=\left(s_{1} s_{2} \ldots\right)$. For an alphabet $\Omega$, a shift space is a subset $X \subset \Omega^{\mathbb{N}}$ which is invariant (with respect to the shift map $\sigma$ ) and compact. In particular, $\Omega^{\mathbb{N}}$ is a shift space. For notes on shift spaces we refer the reader to [10].

Let $I$ be a compact interval and $f: I \rightarrow I$ be continuous. Let $c_{0}<c_{1}<$ $\cdots<c_{l+1} \in I$ be such that $I=\left[c_{0}, c_{l+1}\right]$ and $c_{1}, \ldots, c_{l}$ are the turning points (or critical points) of $f$; we call such maps piecewise monotone, or l-modal. Define the monotone subintervals $I_{i}=\left(c_{i-1}, c_{i}\right)$ for $i=2, \ldots, l, I_{1}=\left[c_{0}, c_{1}\right)$ and $I_{l+1}=\left(c_{l}, c_{l+1}\right]$, and define the alphabet $\Omega=\left\{I_{1}, \ldots, I_{l+1}, C_{1}, \ldots, C_{l}\right\}$. Then let the address map $A: I \rightarrow \Omega$ be defined by

$$
A(x)= \begin{cases}I_{i} & \text { if } x \in I_{i}, 1 \leq i \leq l+1, \\ C_{j} & \text { if } x=c_{j}, 1 \leq j \leq l,\end{cases}
$$

where we abuse notation slightly in the double meaning of $I_{i}$. Now define the itinerary map It $: I \rightarrow \Omega^{\mathbb{N}}$ by

$$
\operatorname{It}(x)=\left(\operatorname{It}_{0}(x) \operatorname{It}_{1}(x) \operatorname{It}_{2}(x) \ldots\right)
$$

where $\mathbf{I t}_{i}(x)=A\left(f^{i}(x)\right)$. Thus the orbit of the point $x$, with respect to the map $f$ is represented symbolically by the itinerary $\operatorname{lt}(x)$ of the point. Define 
the upper and lower limit-itineraries $\operatorname{It}\left(x^{+}\right)$and $\operatorname{It}\left(x^{-}\right)$respectively of the point $x$ as $\operatorname{It}\left(x^{+}\right)=\lim _{y \downarrow x} \operatorname{It}(y)$ and $\operatorname{It}\left(x^{-}\right)=\lim _{y \uparrow x} \operatorname{It}(y)$ (where the limit, which exists by compactness, is taken in $\Omega^{\mathbb{N}}$ ); then the kneading invariants $K_{j}$ for $1 \leq j \leq l$ of the map $f$ are given by $K_{j}=\sigma\left(\operatorname{lt}\left(c_{j}^{+}\right)\right)$.

Let $D f(x)$ be the gradient of $f$ at $x$. The polarity of an element $\alpha \in \Omega$ is given by the map $\rho: \Omega \rightarrow\{-1,+1\}$, where

$$
\rho(\alpha)= \begin{cases}+1 & \text { if either } D f(x)>0 \text { for } x \in \alpha, \text { or } \alpha=C_{j} \text { for some } j, \\ -1 & \text { if } D f(x)<0 \text { for } x \in \alpha .\end{cases}
$$

We say that a finite word $s_{0} \ldots s_{n}$ over $\Omega$ is even if $\prod_{i=0}^{n} \rho\left(s_{i}\right)=1$ and odd otherwise. Finally, define the parity lexicographic ordering, $\prec$, on $\Omega^{\mathbb{N}}$ by declaring $s \prec t$ provided the discrepancy between $s$ and $t$ is $k>0$, and either

- $s \uparrow_{k-1}=t \uparrow_{k-1}$ is even and $s_{k}<t_{k}$, or

- $s \uparrow_{k-1}=t \uparrow_{k-1}$ is odd and $s_{k}>t_{k}$.

2.1 to 2.5 are well known facts about the symbolic dynamics of $l$-modal maps (see for example [6], [7] and [11]). The first shows that the parity lexicographic ordering preserves the ordering on the interval.

Lemma 2.1. For $x, y \in I$, if $\operatorname{lt}(x) \prec \operatorname{lt}(y)$ then $x<y$.

For $x \in I$ we define $I_{N}(x)=\left\{y \in I:\left.\operatorname{lt}(y)\right|_{N}=\operatorname{lt}(x)\left\lceil_{N}\right\}\right.$. We call a point $x \in I$ pre-critical if there is a positive integer $k$ for which $f^{k}(x)=c_{j}$ for $1 \leq j \leq l$.

Lemma 2.2. Let $x \in I$. For each $N \in \mathbb{N}, I_{N}(x)$ is an interval in $I$. Moreover, if $f^{n}(x)=c_{k}$ for some $n \leq N$ and some critical point $c_{k}$, then $I_{N}(x)=\{x\}$, otherwise $I_{N}(x)$ is an open interval.

Lemma 2.3. The itinerary map is discontinuous at $x$ if and only if $x$ is a pre-critical point.

It is clear that any point $x \in I$ has an associated itinerary, but it is not the case that every sequence in $\Omega^{\mathbb{N}}$ is the itinerary of some point. However, we can place conditions on sequences which ensure they correspond to the itinerary of a point in the interval [7].

Definition 2.4 (Admissibility conditions). Let $\Sigma_{K}$ denote the subset of $\Omega^{\mathbb{N}}$ which consists of sequences $\mathbf{s}=\left(s_{0} s_{1} \ldots\right)$ obeying the following conditions. For every $n \geq 0$ and for $1 \leq k \leq l$,

$$
\begin{aligned}
\sigma^{n}(\mathbf{s})=\operatorname{lt}\left(c_{k}\right) & \text { if } s_{n}=C_{k}, \\
K_{k} \prec \sigma^{n+1}(\mathbf{s}) \prec K_{k+1} & \text { if } s_{n}=I_{k+1} \text { and } \rho\left(s_{n}\right)=+1, \\
K_{k+1} \prec \sigma^{n+1}(\mathbf{s}) \prec K_{k} & \text { if } s_{n}=I_{k+1} \text { and } \rho\left(s_{n}\right)=-1,
\end{aligned}
$$


where we ignore the redundant invariants $K_{l+1}$ if $s_{n}=I_{l+1}$, or $K_{0}$ if $s_{n}=I_{1}$. Sequences in $\Sigma_{K}$ will be called admissible. Let $\hat{\Sigma}_{K}$ be defined as $\Sigma_{K}$ but with $\prec$ replaced by $\preceq$ in $(2)$ and (3).

TheOREM 2.5. $\Sigma_{K} \subseteq \operatorname{lt}(I) \subseteq \hat{\Sigma}_{K}$.

Thus a sequence which obeys admissibility conditions (1)-(3) is the itinerary of some point in the interval.

A piecewise monotone map $f: I \rightarrow I$ is called locally eventually onto if for every open interval $U \subset I$ there is a $k=k(U) \in \mathbb{N}$ for which $f^{k}(U)=I$; for this paper we define a similar but weaker condition.

Definition 2.6. An $l$-modal map with critical points $c_{1}, \ldots, c_{l}$ is called locally pre-critical if for every open interval $U \subset I$ there is a $k=k(U) \in \mathbb{N}$ for which $c_{j} \in f^{k}(U)$ for some $1 \leq j \leq l$.

Clearly every map which is locally eventually onto is also locally precritical, but the converse is not true in general. To illustrate these properties we recall an example from [4].

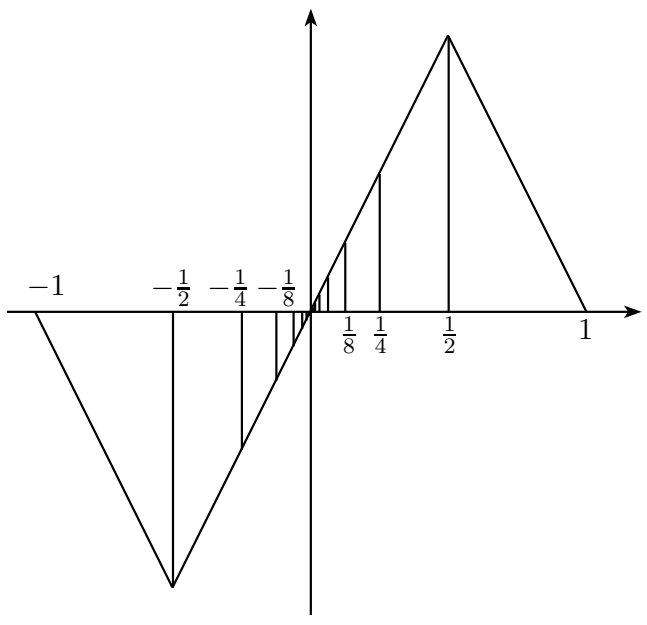

Fig. 1. The dynamics of the double tent map are disjoint about 0 .

Example 2.7. Consider the tent map $f_{2}:[0,1] \rightarrow[0,1]$ defined as

$$
f_{2}(x)= \begin{cases}2 x, & x \in[0,1 / 2], \\ 2(1-x), & x \in[1 / 2,1],\end{cases}
$$

and let us extend this to a map $g:[-1,1] \rightarrow[-1,1]$ defined by

$$
g(x)= \begin{cases}-f_{2}(x), & x \in[-1,0], \\ f_{2}(x), & x \in[0,1]\end{cases}
$$


(see Figure 1). The dynamics for the two halves of this map are disjoint about 0 ; no point in $(0,1]$ is mapped to $[-1,0)$ and vice versa.

Consider the sets

$$
H_{1}=\{0\} \cup \bigcup_{n=0}^{\infty}\left\{\frac{1}{2^{n}}\right\} \text { and } H_{2}=\{0\} \cup \bigcup_{n=0}^{\infty}\left\{-\frac{1}{2^{n}}\right\}
$$

For $x=1 / 2^{i} \in H_{1}$ with $i \geq 0$, it is easy to see that for $k \leq i, g^{k}(x)=1 / 2^{i-k}$, so $H_{1}$ is a backward orbit of the point 0 , as is $H_{2}$ for similar reasons. The map $g$ is locally pre-critical, since any open interval will contain an open interval in either $(0,1)$ or $(-1,0)$, and both $f_{2}$ and $-f_{2}$ are locally eventually onto when restricted to $[0,1]$ and $[-1,0]$ respectively [5]. However, $g$ is not locally eventually onto, since $[0,1]$ and $[-1,0]$ are both invariant under $g$.

This example can also be found in [1], and we use it throughout the following to demonstrate some of the more subtle characteristics of $\omega$-limit sets.

We wish to use the simplicity of the dynamics of the shift map to make deductions on the dynamics of piecewise monotone maps. To this end we require the set of itineraries of points in a subset $L$ of the interval to be a homeomorphic copy of $L$. We show in Theorem 2.9 that this is the case when the map in question is locally pre-critical, but the set itself contains no pre-critical points.

Lemma 2.8. Suppose that $f: I \rightarrow I$ is an l-modal map with critical points $c_{1}, \ldots, c_{l}$ and define $\Gamma=\{\operatorname{lt}(x): x \in I\}$. If $f$ is locally pre-critical then the itinerary map is one-to-one and the inverse itinerary map $\mathrm{It}^{-1}$ : $\Gamma \rightarrow I$ is continuous.

Proof. Let $x, y \in I$ for $x<y$. Then there is an $n \in \mathbb{N}$ for which $c_{k} \in$ $f^{n}((x, y))$ for some $k$, and let $n$ be minimal in this respect. Then for every $i<n, f^{i}((x, y))$ is strictly contained in an interval of monotonicity, so $f^{i}(x) \neq f^{i}(y)$ for all such $i$. Thus $c_{k}$ lies between $f^{n}(x)$ and $f^{n}(y)$, implying $\mathrm{It}_{n}(x) \neq \mathrm{It}_{n}(y)$. Hence $\mathrm{It}$ is one-to-one.

Now pick $s \in \Gamma$ and let $\epsilon>0$. Then $I_{N}(x)=\left\{y \in I: \operatorname{It}(y)\left\lceil_{N}=\operatorname{lt}(x)\left\lceil_{N}\right\}\right.\right.$ is an interval by Lemma 2.2. Since the itinerary map is a bijection onto $\Gamma$ we get that $\bigcap_{N \in \mathbb{N}} I_{N}(x)=\{x\}$, so there is an $N^{*} \in \mathbb{N}$ such that for every $y \in I_{N^{*}}(x)$ we have $|x-y|<\epsilon$. Set $\delta=1 / 2^{N^{*}}$. Then whenever $t \in \Gamma$ is such that $d(s, t)<\delta$, we have that $\mathrm{It}^{-1}(t) \in I_{N^{*}}(x)$ and so $\left|\mathrm{It}^{-1}(t)-\mathrm{It}^{-1}(s)\right|<\epsilon$.

Theorem 2.9. Suppose that for some $l \in \mathbb{N}, f: I \rightarrow I$ is a locally pre-critical, l-modal map with critical points $c_{1}, \ldots, c_{l}$. Suppose also that $L \subset I$ does not contain $c_{k}$ for any $1 \leq k \leq l$ and is such that $f(L) \subset L$; define $\Lambda=\{\operatorname{It}(x): x \in L\}$. Then $\mathrm{It}: L \rightarrow \Lambda$ is a homeomorphism. 
Proof. Since $c_{k} \notin L$ for any $1 \leq k \leq l$, no $x \in L$ is pre-critical since $f(L) \subset L$, so It is continuous on $L$ by Lemma 2.3. Moreover, It : $L \rightarrow \Lambda$ is bijective and $\mathrm{It}^{-1}$ is continuous by Lemma 2.8 .

3. Internal chain transitivity in shift spaces. In this section we use the fact that internal chain transitivity characterizes $\omega$-limit sets in certain shift spaces to construct a sequence containing all finite words from elements in a set $\Lambda$ whose $\omega$-limit set is $\Lambda$ itself. This section contains material which is a reworking of that in [2].

The first result is a fact about $\omega$-limit sets of sequences in shift spaces [8].

Lemma 3.1. Suppose that $s$ and $t$ are sequences in $\Omega^{\mathbb{N}}$. Then $t \in \omega(s, \sigma)$ if and only if every finite initial segment of t occurs infinitely often in $s$.

The next lemma shows how pseudo-orbits appear in shift spaces.

Lemma 3.2. Suppose that $\Omega$ is an alphabet and $\Lambda$ is a subset of $\Omega^{\mathbb{N}}$. For $\epsilon>0$ and $s, t \in \Lambda$, if $\left\{s_{0}=s, s_{1}, \ldots, s_{k}=t\right\}$ is an $\epsilon$-pseudo-orbit joining $s$ and $t$, then for $n \in \mathbb{N}$ with $1 / 2^{n}<\epsilon \leq 1 / 2^{n+1}$, for each $0 \leq i \leq k-1$ the first $n-1$ symbols of $\sigma\left(s_{i}\right)$ agree with the first $n-1$ symbols of $s_{i+1}$.

Proof. We know that for each $0 \leq i \leq k-1$, there is a positive integer $n_{i}$ for which $d\left(\sigma\left(s_{i}\right), s_{i+1}\right)=1 / 2^{n_{i}}<\epsilon$. Thus the first $n_{i}-1$ symbols of $\sigma\left(s_{i}\right)$ and $s_{i+1}$ coincide. Suppose that $n \in \mathbb{N}$ is such that $1 / 2^{n}<\epsilon \leq 1 / 2^{n+1}$. Then $n \leq \min \left\{n_{i}: 0 \leq i \leq k-1\right\}$ and so for each $0 \leq i \leq k-1$ the first $n-1$ symbols of $\sigma\left(s_{i}\right)$ agree with the first $n-1$ symbols of $s_{i+1}$.

Suppose that $\Omega$ is an alphabet, $N$ is a positive integer and $\Lambda$ is a nonempty, internally chain transitive subset of $\Omega^{\mathbb{N}}$. Let $\mathcal{A}$ be the collection of all finite words of length greater than $N$ which appear in elements of $\Lambda$, and enumerate $\mathcal{A}$ as $\left\{\theta_{n}\right\}$. For every $n \in \mathbb{N}$ pick $q_{n}, q_{n+1} \in \Lambda$ such that $\theta_{n}$ is the initial segment of $q_{n}$ and $\theta_{n+1}$ is the initial segment of $q_{n+1}$. Also for each $n \in \mathbb{N}$ pick $m_{n}>\max \left\{\left|\theta_{n}\right|,\left|\theta_{n+1}\right|\right\}$ and let $\epsilon=1 / 2^{m_{n}+1}$. Then since $\Lambda$ is internally chain transitive there is an $\epsilon$-pseudo-orbit of elements from $\Lambda$ joining $q_{n}$ and $q_{n+1}$. By Lemma 3.2 , for each $n \in \mathbb{N}$ we have points $q_{n, 0}=q_{n}, q_{n, 1}, \ldots, q_{n, k_{n}}=q_{n+1} \in \Lambda$ such that for $1 \leq i \leq k_{n}$, the first $m_{n}-1$ symbols of $\sigma\left(q_{n, i-1}\right)$ agree with the first $m_{n}-1$ symbols of $q_{n, i}$.

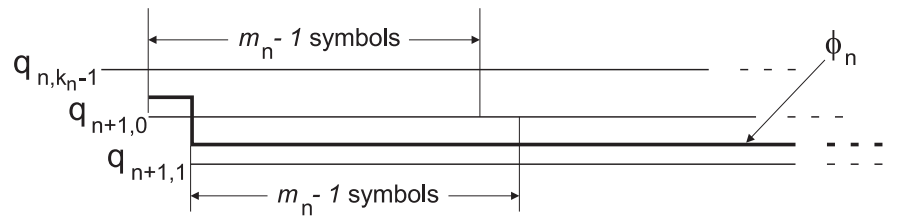

Fig. 2. The relationship between the various sequences in the construction of $s(\Lambda, N)$. 
We construct an element $s(\Lambda, N) \in \Omega^{\mathbb{N}}$ as follows: For every $n \in \mathbb{N}$ we make a new word $\phi_{n}$ from $\theta_{n}, \theta_{n+1}$ and the $\epsilon$-pseudo-orbit joining the corresponding $q_{n}, q_{n+1}$, by taking the first symbol of each $q_{n, i}$ and constructing $\phi_{n}$ sequentially from these. So $\phi_{n}$ begins with an initial segment of $q_{n-1, k_{n-1}}=q_{n, 0}$ (the first section of which is $\theta_{n}$ ) and ends with the first symbol of $q_{n, k_{n}-1}$; then $\phi_{n+1}$ begins with an initial segment of $\sigma\left(q_{n, k_{n}-1}\right)=q_{n+1,0}$ (see Figure 2). The sequence $s(\Lambda, N)$ is then the concatenation of all the $\phi_{n}$. Notice that for each $i$, the agreement between $\sigma\left(q_{n, i}\right)$ and $q_{n, i+1}$ is at least as long as each of $\theta_{n}$ and $\theta_{n+1}$.

Definition 3.3. We call the element $s(\Lambda, N)$ in the above construction an arbitrary length, infinite repetition sequence for the internally chain transitive set $\Lambda$.

Lemma 3.4. Suppose that $\Omega$ is an alphabet and $\Lambda \subset \Omega^{\mathbb{N}}$ is a shift space which is internally chain transitive. If for some $N \in \mathbb{N}, s=s(\Lambda, N)$ is an arbitrary length, infinite repetition sequence for $\Lambda$, then $\Lambda=\omega(s, \sigma)$.

Proof. Let $\mathcal{A}$ be the set of all finite words of length greater than $N$ which occur in elements of $\Lambda$. Then since $\Lambda$ is invariant, $\mathcal{A}$ is also the set of all finite words of length greater than $N$ which occur as initial segments of elements of $\Lambda$. We first show that $\mathcal{A}$ is the set of all infinitely repeating words of length greater than $N$ occurring in $s$.

Let $V \in \mathcal{A}$ be a word of length greater than $N$. Then $V$ occurs as a subword infinitely often in $\mathcal{A}$, and hence by construction infinitely often in $s$. Now suppose that the finite word $V$ has length greater than $N$ and occurs infinitely often in $s$. By the construction of $s$, there is an $n \in \mathbb{N}$ for which all of the words from $\mathcal{A}$ occurring in $\sigma^{n}(s)$ are of length greater than that of $V$, so pick an occurrence of $V$ in $\sigma^{n}(s)$. Since for every $k \in \mathbb{N}, \sigma^{k}(s)$ begins with a segment of some point in $\Lambda$ (see Figure 2), this $V$ must be the initial segment of some point in $\Lambda$, so must also be the initial segment of some word from $\mathcal{A}$, and since $\mathcal{A}$ is invariant under taking subwords of length greater than $N$, we must have $V \in \mathcal{A}$.

Pick $t \in \Lambda$. Then every finite initial segment of $t$ of length greater than $N$ is in $\mathcal{A}$, so occurs infinitely often in $s$, and hence by Lemma 3.1, $t \in \omega(s, \sigma)$. Now pick $t \in \omega(s, \sigma)$. Then by Lemma 3.1 every finite initial segment of $t$ of length greater than $N$ occurs infinitely often in $s$, and so is in $\mathcal{A}$. As $\Lambda$ is closed, $t \in \Lambda$ (since $t$ is the limit of points in $\Lambda$ with initial segments of increasing length which agree with those of $t$ ). Hence $\Lambda=\omega(s, \sigma)$ as required.

4. $\omega$-Limit sets of piecewise monotone maps. In this section we prove our main theorem, which places conditions upon subsets of the interval 
under which internal chain transitivity characterizes $\omega$-limit sets of locally pre-critical, piecewise monotone maps.

The first lemma shows that properties of certain subsets of the interval are retained by their images under the itinerary map.

Lemma 4.1. Suppose that for some $l \in \mathbb{N}, f: I \rightarrow I$ is a locally precritical, l-modal map with critical points $c_{1}, \ldots, c_{l}$, and that $L \subset I$ is such that $c_{k} \notin L$ for any $1 \leq k \leq l$. Let $\Lambda=\operatorname{lt}(L)$. Then $L$ is closed, invariant, non-empty and internally chain transitive if and only if $\Lambda$ is closed, invariant, non-empty and internally chain transitive.

Proof. Suppose that $L$ is closed, invariant, non-empty and internally chain transitive. Then clearly $\Lambda$ is non-empty. By Theorem 2.9 we know that It : $L \rightarrow \Lambda$ is continuous; $L$ is compact so $\Lambda$ is compact, and thus closed in the Hausdorff space $\Omega^{\mathbb{N}}$. Moreover, $\sigma \circ \mathrm{lt}=\mathrm{It} \circ f$, so $\Lambda$ is invariant.

To show that $\Lambda$ is internally chain transitive, pick $r, s \in \Lambda$, where $r=$ $\operatorname{lt}(y)$ and $s=\operatorname{lt}(x)$ for some $x, y \in L$, and let $\epsilon>0$. We need a sequence $\left\{s=s_{0}, s_{1}, \ldots, s_{n}=r\right\} \subset \Lambda$ for which $d\left(\sigma\left(s_{i-1}\right), s_{i}\right)<\epsilon$ for every $1 \leq i \leq n$. Now It $: L \rightarrow \Lambda$ is continuous, so is also uniformly continuous since $L$ is compact. So there is a $\delta>0$ such that for every $x, y \in L$ for which $|x-y|<\delta$ we have $d(\operatorname{lt}(x), \operatorname{lt}(y))<\epsilon$. Since $L$ is internally chain transitive there exist $x_{0}=x, x_{1}, \ldots, x_{n}=y$ for which $\left|f\left(x_{i-1}\right)-x_{i}\right|<\delta$ for every $1 \leq i \leq n$. Hence $d\left(\operatorname{It}\left(f\left(x_{i-1}\right)\right), \operatorname{lt}\left(x_{i}\right)\right)<\epsilon$. Thus, setting $s_{i}=\operatorname{lt}\left(x_{i}\right)$ and noting that by conjugation $\operatorname{It}\left(f\left(x_{i-1}\right)\right)=\sigma\left(\operatorname{It}\left(x_{i-1}\right)\right)$, we conclude that $d\left(\sigma\left(s_{i-1}\right), s_{i}\right)<\epsilon$ for every $1 \leq i \leq n$. The converse is identical.

Proposition 4.2. Suppose that $f: I \rightarrow I$ is a locally pre-critical, $l$ modal map with critical points $c_{1}, \ldots, c_{l}$, and that $L \subset I$ is closed, invariant, non-empty and does not contain $c_{j}$ for any $1 \leq j \leq l$. Suppose also that for $x_{0} \in I,\left\{f^{n}\left(x_{0}\right): n \in \mathbb{N}\right\}$ is bounded away from $c_{j}$ for each $1 \leq j \leq l$. Then $\operatorname{lt}(L)=\omega\left(\operatorname{lt}\left(x_{0}\right), \sigma\right)$ if and only if $L=\omega\left(x_{0}, f\right)$.

Proof. Since $\left\{f^{n}\left(x_{0}\right): n \in \mathbb{N}\right\}$ is bounded away from $c_{j}$ for $1 \leq j \leq l$, It is a homeomorphism on $\overline{\left\{f^{n}\left(x_{0}\right): n \in \mathbb{N}\right\}}$ by Theorem 2.9. So

$$
\begin{aligned}
\omega\left(\operatorname{It}\left(x_{0}\right), \sigma\right) & =\bigcap_{n \geq 0} \overline{\left\{\sigma^{k}\left(\operatorname{It}\left(x_{0}\right)\right): k>n\right\}} \\
& =\bigcap_{n \geq 0} \overline{\left\{\operatorname{lt}\left(f^{k}\left(x_{0}\right)\right): k>n\right\}} \\
& =\bigcap_{n \geq 0} \operatorname{It}\left(\overline{\left\{f^{k}\left(x_{0}\right): k>n\right\}}\right) \quad \text { (since It is continuous) }
\end{aligned}
$$




$$
\begin{aligned}
& =\operatorname{It}\left(\bigcap_{n \geq 0} \overline{\left\{f^{k}\left(x_{0}\right): k>n\right\}}\right) \quad \text { (since It is injective) } \\
& =\operatorname{It}\left(\omega\left(x_{0}, f\right)\right) .
\end{aligned}
$$

Suppose that $\operatorname{It}(L)=\omega\left(\operatorname{It}\left(x_{0}\right), \sigma\right)$. Then by the above calculation, $\operatorname{It}(L)=$ It $\left(\omega\left(x_{0}, f\right)\right)$. As It is a homeomorphism on $L$ by Theorem 2.9, it is certainly injective, hence we must have $L=\omega\left(x_{0}, f\right)$.

Now suppose that $L=\omega\left(x_{0}, f\right)$. Then clearly $\operatorname{lt}(L)=\operatorname{lt}\left(\omega\left(x_{0}, f\right)\right)$, i.e. $\operatorname{It}(L)=\omega\left(\operatorname{It}\left(x_{0}\right), \sigma\right)$, again by the above calculation.

We can now prove our main result.

THEOREM 4.3. Suppose that $f: I \rightarrow I$ is a locally pre-critical, l-modal map with critical points $c_{1}, \ldots, c_{l}$, and that $L \subset I$ is a closed, invariant, non-empty set which does not contain $f\left(c_{j}\right)$ for any $1 \leq j \leq l$. Then $L$ is internally chain transitive if and only if $L=\omega\left(x_{0}, f\right)$ for some $x_{0} \in I$.

Proof. Suppose that $L=\omega\left(x_{0}, f\right)$. Then $L$ is known to be internally chain transitive. Now suppose that $L \subset I$ is a closed, invariant and nonempty set which does not contain the image of any critical point of $f$, and thus none of the critical points themselves, and is internally chain transitive. Then by Lemma 4.1. $\Lambda=\{\operatorname{lt}(x): x \in L\}$ is closed, invariant, non-empty and internally chain transitive. Since $f\left(c_{j}\right) \notin L$ for any $1 \leq j \leq l$ and $L$ is closed it must be bounded away from each $f\left(c_{j}\right)$, so by uniform continuity of $\mathrm{It}^{-1}$, $\Lambda$ must be bounded away from each $K_{j}$. Thus for every $1 \leq j \leq l$ there is an $N_{j} \in \mathbb{N}$ such that the discrepancy between $t$ and $K_{j}$ is less than $N_{j}$ for every $t \in \Lambda$; let $N=\max \left\{N_{j}: 1 \leq j \leq l\right\}$. Now construct an arbitrary length, infinite repetition sequence $s=s(\Lambda, N)$. By Lemma 3.4, $\Lambda=\omega(s, \sigma)$.

We want to have $s=\operatorname{It}\left(x_{0}\right)$ for some $x_{0} \in[0,1]$, so we show that the admissibility conditions (1)-(3) in Definition 2.4 are satisfied by $s$. By the construction of $s$, for any $k \in \mathbb{N}, \sigma^{k}(s)$ begins with at least the first $N$ symbols of some $t \in \Lambda$, within which we can see a discrepancy with every kneading invariant $K_{j}$, as noted above (this implies that for any $k \in \mathbb{N}$, $\sigma^{k}(s)$ is bounded away from each $\left.K_{j}\right)$. Since each such $t$ is the itinerary of some point in $L$, this discrepancy tells us that $\sigma^{k}(s)$ satisfies conditions (1) - (3). Thus by Theorem 2.5, there is an $x_{0} \in I$ for which $s=\operatorname{lt}\left(x_{0}\right)$. Hence $\Lambda=\omega\left(\operatorname{It}\left(x_{0}\right), \sigma\right)$. Moreover, since $\sigma^{k}(s)$ is bounded away from each $K_{j}$ for every $k \in \mathbb{N}$, and It is uniformly continuous, it follows that $f^{k}\left(x_{0}\right)$ is bounded away from $c_{j}$ for each $1 \leq j \leq l$ and for every $k \geq 0$. So by Proposition 4.2 we have $L=\omega\left(x_{0}, f\right)$ as required.

REMARK 4.4. It may appear that the condition of $\Lambda$ not containing the image of any critical point is simply an artefact of using symbolic dynamics. However, in Example 2.7 the set $H=H_{1} \cup H_{2}$, which contains the image of 
both critical points, is a closed, invariant, non-empty set which is internally chain transitive but not the $\omega$-limit set of any point.

To demonstrate that there are indeed $\omega$-limit sets of locally pre-critical maps of the interval (other than periodic orbits) which do not contain any critical point or image thereof, we refer to Good and Raines [8], who give examples of uncountably many locally pre-critical tent maps whose postcritical $\omega$-limit sets are Cantor sets and do not contain the critical point or its image.

However, Theorem 4.3 does not characterize all $\omega$-limit sets of locally pre-critical, piecewise monotone maps of the interval. In the next section we identify an $\omega$-limit set for such a map which does contain a critical point.

5. Identifying $\omega$-limit sets using symbolic dynamics. In this section we demonstrate how the symbolic dynamics can be used to show that one point in the interval is in the $\omega$-limit set of another. Using this we find an $\omega$-limit set of a piecewise monotone map of a compact interval which contains a critical point.

We begin by proving three lemmas relating to limit-itineraries of points in the interval.

Lemma 5.1. Suppose that the l-modal map $f: I \rightarrow I$ is locally precritical. Then $x>y$ implies that $\operatorname{lt}(x) \succeq \operatorname{lt}\left(x^{-}\right) \succ \operatorname{lt}\left(y^{+}\right) \succeq \operatorname{lt}(y)$.

Proof. It is clear from the definition of the limit-itineraries that $\operatorname{lt}(x) \succeq$ $\operatorname{It}\left(x^{-}\right)$and $\operatorname{It}\left(y^{+}\right) \succeq \operatorname{It}(y)$. Moreover, by Lemma 2.1 and the fact that the itinerary map is bijective, every $z \in(y, x)$ is such that $\operatorname{lt}\left(x^{-}\right) \succ \operatorname{lt}(z) \succ$ It $\left(y^{+}\right)$.

Lemma 5.2. Let $f: I \rightarrow I$ be an l-modal map.

(1) If $f^{i}(x) \neq c_{k}$ for any $i$ and for any $1 \leq k \leq l$ then $\operatorname{lt}(x)=\operatorname{lt}\left(x^{+}\right)=$ It $\left(x^{-}\right)$.

(2) If $f^{n}(x)=c_{k}$ for some $n \in \mathbb{N}$ and some $1 \leq k \leq l$, then for small enough $\delta$,

(a) $\operatorname{It}_{n}(y)=I_{k+1}$ for all $y \in(x, x+\delta)$ and $\mathbf{I t}_{n}(y)=I_{k}$ for all $y \in(x-\delta, x)$ if $\operatorname{It}(y) \uparrow_{n-1}$ is even,

(b) $\mathrm{It}_{n}(y)=I_{k}$ for all $y \in(x, x+\delta)$ and $\mathbf{I t}_{n}(y)=I_{k+1}$ for all $y \in(x-\delta, x)$ if $\left.\operatorname{lt}(y)\right|_{n-1}$ is odd.

For the least such $n$ we have $\operatorname{It}(x) \uparrow_{n-1}=\operatorname{It}\left(x^{+}\right) \Gamma_{n-1}=\operatorname{lt}\left(x^{-}\right) \Gamma_{n-1}$.

(3) For every $x \in I, C_{k}$ does not appear in $\operatorname{lt}\left(x^{+}\right)$or $\operatorname{It}\left(x^{-}\right)$for any $1 \leq k \leq l$.

Proof. Let $x \in I$ be fixed. Notice that by the continuity of $f$, for any $n \in \mathbb{N}$ there is a $\delta>0$ for which $\operatorname{lt}_{n}(y)$ is constant on $(x, x+\delta)$ and 
$(x-\delta, x)$, although if $\operatorname{It}_{n}(x)=C_{k}$ for some $1 \leq k \leq l$ then if $\left.\operatorname{tt}(x)\right|_{n-1}$ is even, $\operatorname{It}_{n}(y)=I_{k+1}$ for all $y \in(x, x+\delta)$ and $\operatorname{It}_{n}(y)=I_{k}$ for all $y \in(x-\delta, x)$ by the definition of the ordering $\prec$ (and vice versa if $\operatorname{It}(x) \uparrow_{n-1}$ is odd). This is because by Lemma 2.2 , when $\operatorname{It}_{n}(x)=C_{k}, I_{N}(x)=\{x\}$ for every $N \geq n$. Thus $C_{k}$ does not appear in $\operatorname{lt}\left(x^{+}\right)$or $\operatorname{lt}\left(x^{-}\right)$for any $1 \leq k \leq l$.

Now suppose that $f^{n}(x)=c_{k}$ for some $n \in \mathbb{N}$ and some $1 \leq k \leq l$, and that $n$ is minimal in this respect. Then for all $i<n$ there is a $\delta>0$ for which $\mathrm{It}_{i}(y)=\mathrm{It}_{i}\left(y^{+}\right)$on $(x, x+\delta)$ and $\mathrm{It}_{i}(y)=\mathrm{It}_{i}\left(y^{-}\right)$on $(x-\delta, x)$. In particular, $\operatorname{It}(x) \Upsilon_{n-1}=\operatorname{It}\left(x^{+}\right) \Gamma_{n-1}=\operatorname{It}\left(x^{-}\right) \Upsilon_{n-1}$. Now suppose that $f^{i}(x) \neq c_{k}$ for all $i \in \mathbb{N}$ and all $1 \leq k \leq l$; this must mean that $\operatorname{It}(x)=\operatorname{It}\left(x^{+}\right)=\operatorname{lt}\left(x^{-}\right)$by the continuity of It.

Lemma 5.3. Suppose that the l-modal map $f: I \rightarrow I$ with critical points $c_{1}, \ldots, c_{l}$ is locally pre-critical, and let $y \in I$. For every $\epsilon>0$ there is a positive integer $N(\epsilon)$ such that for every $x \in I$ which satisfies $\operatorname{lt}(x) \Gamma_{N(\epsilon)}=$ $\operatorname{lt}\left(y^{+}\right) \Gamma_{N(\epsilon)}$ or $\operatorname{lt}(x) \Gamma_{N(\epsilon)}=\operatorname{lt}\left(y^{-}\right)\left\lceil_{N(\epsilon)}\right.$ we have $x \in(y-\epsilon, y+\epsilon)$.

Proof. Case 1: suppose that there is no $k \in \mathbb{N}$ such that $f^{k}(y)=c_{j}$ for any $1 \leq j \leq l$. Thus $\operatorname{lt}\left(y^{+}\right)=\operatorname{lt}(y)=\operatorname{It}\left(y^{-}\right)$and the itinerary map is continuous at $y$ by Lemma 2.3. Assume the lemma is false, i.e. that there is an $\epsilon>0$ such that for every $n \in \mathbb{N}$ there is a point $z_{n} \in I$ such that $\operatorname{It}\left(z_{n}\right)$ and $\operatorname{It}(y)$ agree up to their first $n$ places, but $z_{n} \notin(y-\epsilon, y+\epsilon)$. Certainly $\lim _{n \rightarrow \infty} \operatorname{It}\left(z_{n}\right)=\operatorname{It}(y)$. Since $I$ is compact, $\lim _{n \rightarrow \infty} z_{n}$ exists, and since It is continuous at $y$ we have $\operatorname{It}\left(\lim _{n \rightarrow \infty} z_{n}\right)=\operatorname{lt}(y)$. Because the itinerary map is bijective we conclude that $\lim _{n \rightarrow \infty} z_{n}=y$, which contradicts our assumption that the lemma is false.

Case 2: suppose that there is a $k \in \mathbb{N}$ for which $f^{k}(y)=c_{j}$ for some $1 \leq j \leq l$, and $k$ is minimal in this respect. Thus by Lemma 5.2, the discrepancy between $\operatorname{It}(y)$ and $\operatorname{It}\left(y^{+}\right)$is $k$, as it is between $\operatorname{It}(y)$ and $\operatorname{It}\left(y^{-}\right)$. Assume that the lemma is false for $\operatorname{lt}\left(y^{+}\right)$, i.e. that there is an $\epsilon>0$ such that for every $n \in \mathbb{N}$ there is a point $z_{n} \in I$ such that $\operatorname{It}\left(z_{n}\right)$ and $\operatorname{It}\left(y^{+}\right)$agree up to their first $n$ places, but $z_{n} \notin(y-\epsilon, y+\epsilon)$ (the proof is analogous for $\left.\operatorname{It}\left(y^{-}\right)\right)$. Pick $z \in(y, y+\epsilon)$. Then since $f$ is locally pre-critical, $\operatorname{lt}(z) \succ \operatorname{lt}\left(y^{+}\right)$ by Lemma 5.1; let their discrepancy be $m$. Let $m^{*}=\max \{m, k\}$. Then by assumption there is a $z_{m^{*}} \in I$ such that $\left.\operatorname{It}\left(z_{m^{*}}\right)\right|_{m^{*}}=\left.\operatorname{It}\left(y^{+}\right)\right|_{m^{*}}$ and $\left|z_{m^{*}}-y\right| \geq \epsilon$. We must have $z_{m^{*}}>y$ by the agreement of $\operatorname{It}\left(z_{m^{*}}\right)$ with $\operatorname{lt}\left(y^{+}\right)$past the discrepancy with $\operatorname{It}(y)$, hence $z_{m^{*}}>y+\epsilon>z$. So we have $y<z<z_{m^{*}}$ with

$$
\operatorname{It}(z) \Upsilon_{m^{*}} \succ \operatorname{tt}\left(y^{+}\right) \Upsilon_{m^{*}}=\operatorname{It}\left(z_{m^{*}}\right) \uparrow_{m^{*}},
$$

which contradicts Lemma 2.1 .

We would like to have an analogue of Lemma 3.1 to enable us to tell when a point $y \in I$ is in the $\omega$-limit set of a point $x$, but it is clear that 
when moving between the interval and the sequence space, problems occur when a critical point is in the forward orbit of a point under consideration. But using instead the limit-itineraries of points and ensuring that the map is locally pre-critical allows us to prove the following result (which appears to be new).

TheOREM 5.4. Suppose that the l-modal map $f: I \rightarrow I$ is locally precritical. Then for $x, y \in I, y \in \omega(x, f)$ if and only if arbitrarily long initial segments of either $\operatorname{lt}\left(y^{+}\right)$or $\operatorname{lt}\left(y^{-}\right)$occur infinitely often in $\operatorname{lt}(x)$.

Proof. Suppose that for $x, y \in I$, arbitrarily long initial segments of It $\left(y^{+}\right)$occur infinitely often in $\mathrm{It}(x)$ (the proof for $\mathrm{It}\left(y^{-}\right)$is analogous), and let $\epsilon>0$. By Lemma 5.3 there is an $N(\epsilon) \in \mathbb{N}$ such that for every $z \in I$ which satisfies $\mathrm{It}(z) \uparrow_{N(\epsilon)}=\operatorname{It}\left(y^{+}\right) \uparrow_{N(\epsilon)}$ we have $z \in(y-\epsilon, y+\epsilon)$. By assumption there is a $k \in \mathbb{N}$ for which $\sigma^{k}(\operatorname{It}(x)) \uparrow_{N(\epsilon)}=\operatorname{lt}\left(y^{+}\right) \uparrow_{N(\epsilon)}$; so $\operatorname{lt}\left(f^{k}(x)\right) \uparrow_{N(\epsilon)}=$ $\left.\operatorname{It}\left(y^{+}\right)\right|_{N(\epsilon)}$ and thus $\left|f^{k}(x)-y\right|<\epsilon$. Since $\epsilon$ was arbitrary, $y \in \omega(x, f)$.

Now suppose that $y \in \omega(x, f)$ and pick $n \in \mathbb{N}$. We want to show that either $\left.\operatorname{It}\left(y^{+}\right)\right|_{n}$ or $\operatorname{It}\left(y^{-}\right) \Gamma_{n}$ occurs infinitely often in $\operatorname{It}(x)$. By Lemma 5.2, for any $i \in \mathbb{N}$ there is a $\delta_{i}>0$ such that $\operatorname{lt}_{i}(x)$ is constant on $\left(y, y+\delta_{i}\right)$ and $\left(y-\delta_{i}, y\right)$. Let $\delta=\min \left\{\delta_{i}: i \leq n\right\}$. Then $\left.\operatorname{It}(z)\right|_{n}=\left.\operatorname{It}\left(y^{+}\right)\right|_{n}$ for all $z \in(y, y+\delta)$ and $\operatorname{It}(z) \Gamma_{n}=\left.\operatorname{It}\left(y^{-}\right)\right|_{n}$ for all $z \in(y-\delta, y)$. There are infinitely many $k \in \mathbb{N}$ for which $\left|f^{k}(x)-y\right|<\delta$ so there are infinitely many $k$ for which either $f^{k}(x) \in(y, y+\delta)$ or $f^{k}(x) \in(y-\delta, y)$. Thus for these infinitely many $k$ either $\operatorname{It}\left(f^{k}(x)\right) \Gamma_{n}=\operatorname{It}\left(y^{+}\right) \Upsilon_{n}$ or $\operatorname{It}\left(f^{k}(x)\right) \Gamma_{n}=\operatorname{It}\left(y^{-}\right) \Gamma_{n}$.

We now return to Example 2.7 and show that the set $H_{1}$ is an $\omega$-limit set for the map $f_{2}$, and hence for $g$ since the dynamics are disjoint about 0 . Since the dynamics are identical on $[-1,0]$ and disjoint from those on $[0,1]$, we also see that $H_{2}$ is an $\omega$-limit set for the map $-f_{2}$, and thus for $g$. In this example, when describing itineraries of points we use a shorthand, where for example $10^{i}$ means 1 followed by $i$ repetitions of a 0 , and $0^{\infty}$ means infinitely many 0's.

Example 5.5. Consider the tent map $f_{2}$ with gradient 2 as defined in Example 2.7 and the set

$$
H_{1}=\{0\} \cup \bigcup_{n=0}^{\infty}\left\{\frac{1}{2^{n}}\right\}
$$

(see Figure 1). The symbolic dynamics can be defined over the set $\Omega=$ $\{0,1, C\}$ where $A(x)=0$ if $x \in[0,1 / 2), A(x)=C$ if $x=1 / 2$ and $A(x)=1$ if $x \in(1 / 2,1]$.

For a point $x=1 / 2^{i+1} \in H_{1}$ for any $i \geq 0$, notice that $x$ is mapped to $1 / 2^{i}$, then $1 / 2^{i-1}$ and so on until it is mapped onto 1 , then onto the fixed point 0 . Hence for such an $x$ we have $\operatorname{It}(x)=\left(0^{i} C 10^{\infty}\right)$ and $\operatorname{It}\left(x^{+}\right)=$ 
$\left(0^{i} 110^{\infty}\right)$. The only points in $[0,1]$ not of this form are 1 which has itinerary $\left(10^{\infty}\right)$ and 0 which has itinerary $\left(0^{\infty}\right)$. Thus this map has kneading invariant $K=\sigma\left(\operatorname{lt}\left(c^{+}\right)\right)=\left(10^{\infty}\right)$.

Consider the sequence $\mathbf{s}=\left(0110^{2} 110^{3} 110^{4} 11 \ldots\right)$, which is the itinerary of a point $y_{0} \in[0,1]$ by Theorem 2.5. Notice that for any $x \in H_{1}$, arbitrarily long initial segments of $\operatorname{It}\left(x^{+}\right)$occur infinitely often in $\operatorname{It}\left(y_{0}\right)=\mathbf{s}$. Hence by Theorem 5.4, $H_{1} \subseteq \omega\left(y_{0}, f_{2}\right)$. Now suppose that $x \notin H_{1}$. Then It $(x+)$ and It $\left(x^{-}\right)$must contain a word of the form $10^{n} 1$, for some $n \in \mathbb{N}$, or the word 111 , neither of which appears infinitely often in It $\left(y_{0}\right)$. So again by Theorem 5.4. $x \notin \omega\left(y_{0}, f_{2}\right)$ for any such $x$. Hence $H_{1}=\omega\left(y_{0}, f_{2}\right)$.

REMARK 5.6. A map $f$ on a compact metric space $(X, d)$ has the pseudoorbit shadowing property if for every $\epsilon>0$ there is a $\delta>0$ such that for every $\delta$-pseudo-orbit $\left\{x_{0}, x_{1}, \ldots\right\}$ there is an $x \in X$ for which $d\left(f^{i}(x), x_{i}\right)<\epsilon$ (we say that $x \epsilon$-shadows $\left\{x_{0}, x_{1}, \ldots\right\}$ ). Notice that the map $g$ in Example 5.5 does not have the pseudo-orbit shadowing property. Indeed, there are pseudo-orbits for $g$ which enter both $[-1,0)$ and $(0,1]$ and which clearly cannot be $\epsilon$-shadowed by any $x \in[-1,1]$. In a later paper we hope to explore the relationship between pseudo-orbit shadowing on sets without critical points and $\omega$-limit sets further.

Acknowledgements. The author gratefully acknowledges the referee's helpful comments pertaining to the first draft of this paper.

\section{References}

[1] F. Balibrea and C. La Paz, A characterization of the $\omega$-limit sets of interval maps, Acta Math. Hungar. 88 (2000), 291-300.

[2] A. D. Barwell, C. Good, R. Knight and B. E. Raines, A characterization of $\omega$-limit sets in shift spaces, Ergodic Theory Dynam. Systems 30 (2010), 21-31.

[3] L. S. Block and W. A. Coppel, Dynamics in One Dimension, Lecture Notes in Math. 1513, Springer, Berlin, 1992.

[4] A. Blokh, A. M. Bruckner, P. D. Humke, and J. Smítal, The space of $\omega$-limit sets of a continuous map of the interval, Trans. Amer. Math. Soc. 348 (1996), 1357-1372.

[5] K. M. Brucks and B. Diamond, A symbolic representation of inverse limit spaces for a class of unimodal maps, in: Continua (Cincinnati, OH, 1994), Lecture Notes in Pure Appl. Math. 170, Dekker, New York, 1995, 207-226.

[6] P. Collet and J.-P. Eckmann, Iterated Maps on the Interval as Dynamical Systems, Progr. Phys. 1, Birkhäuser, Boston, 1980.

[7] W. de Melo and S. van Strien, One-Dimensional Dynamics, Ergeb. Math. Grenzgeb. 25, Springer, Berlin, 1993.

[8] C. Good and B. E. Raines, Continuum many tent map inverse limits with homeomorphic postcritical $\omega$-limit sets, Fund. Math. 191 (2006), 1-21.

[9] M. W. Hirsch, L. S. Hal and X.-Q. Zhao, Chain transitivity, attractivity, and strong repellors for semidynamical systems, J. Dynam. Differential Equations 13 (2001), 107-131. 
[10] D. Lind and B. Marcus, An Introduction to Symbolic Dynamics and Coding, Cambridge Univ. Press, Cambridge, 1995.

[11] J. Milnor and W. Thurston, On iterated maps of the interval, in: Dynamical Systems (College Park, MD, 1986-87), Lecture Notes in Math. 1342, Springer, Berlin, 1988, $465-563$.

Andrew D. Barwell

School of Mathematics and Statistics

University of Birmingham

Birmingham, B15 2TT, UK

E-mail: barwella@for.mat.bham.ac.uk

Received 6 April 2009;

in revised form 22 September 2009 